\title{
Forelimb Amputation due to Radial Osteosarcoma in a Chinkara Deer
} (Gazella bennettii) in Pakistan

\author{
Nasir Iqbal $^{1 *}$, Naveed Hussain ${ }^{1}$, Sadaf Aslam ${ }^{1}$ and Hamza Jawad ${ }^{2}$ \\ ${ }^{1}$ Department of Veterinary Surgery and Pet Sciences, University of Veterinary \\ and Animal Sciences, Lahore, Punjab, Pakistan \\ ${ }^{2}$ University College of Veterinary and Animal Sciences, The Islamia University of \\ Bahawalpur, Bahawalpur, Punjab, Pakistan
}

Received: April 19, 2020

Published: May 29, 2020

(c) All rights are reserved by Nasir Iqbal., et al.

*Corresponding Author: Nasir Iqbal, Department of Veterinary Surgery and Pet

Sciences, University of Veterinary and Animal Sciences, Lahore, Punjab, Pakistan.

\begin{abstract}
Osteosarcoma (OSA) is the most pervasive bone tumor in the skeletal framework. Metaphyseal region of the appendicular skeleton is the most normally influenced site by OSA especially in more established age animals. The long bones explicitly the radius has been seen to be the most frequently influenced site. Seven years old male Chinkara deer (Gazella bennettii) was referred to clinic for evaluation of the chronic left thoracic limb lameness and progressively increasing pain on the palpation site below and at level of the elbow joint. Patient's overall health status was good. Upon Physical examination marked painful swelling was observed below and at level of elbow joint. Radiographic examination revealed large extra-osseous mass/growth originating from distal extremity of radius extending up to the elbow joint. Based on history, physical examination and radiographic examination case was diagnosed as radial osteosarcoma. Hence left thoracic limb amputation was performed. Upon examination it was observed that about 90 percent of radius was involved, so mid-humeral amputation was performed.

Keywords: Deer; Osteosarcoma; Radius; Amputation; Mid Humeral
\end{abstract}

\section{Introduction}

Osteosarcoma is the most typical bone tumor emerging from the malignant osteoblasts or stem cells. (Approximately $98 \%$ of neoplasms of bone are malignant in nature. Out of these $85 \%$ of cases involve Osteosarcomas) [1]. OSA spreads rapidly to involve the surrounding soft tissues causing severe pain at the affected site. As OSA progresses it results in the decrease in bone strength and in severe cases it may lead to fracture of affected bone [2]. OSA most commonly affects the metaphyseal region of the bones (specifically long and flat bones) in the appendicular skeleton. The incidence of appendicular OSA is higher in middle to old aged animals, large/giant breeds and animals with higher growth rate. OSA's have a much higher tendency to metastasize to the soft tissues most commonly lungs are involved (Pulmonary Metastasis) [3-8].

Limb amputation is considered as a gold standard treatment for the osteosarcomas. (Though limb sparing techniques are also being adopted). Limb amputation surgery has very less chances of tumor recurrence as compared to the Limb sparing techniques (which contains risks of infection, tumor recurrence and implant loosening or failure). The present paper describes successful management of the radial OSA in a chinkara deer [4-9].

\section{Case History}

A 7 years old Chinkara deer weighing $20 \mathrm{~kg}$ was presented at the clinic with the history of chronic lameness and pain in the right forelimb. Upon Clinical examination, firm and painful swelling was observed from elbow joint till the distal radial extremity. Then left forelimb radiography was performed which showed medullary and cortical bone destruction, periosteal bone proliferation and Codman triangle formation. Soft Tissue swelling was also observed at the site of affected bone. Thoracic radiographs were taken to check the metastasis and no pulmonary metastasis was seen in this case. Clinical examination and radiographic features were so characteristic of the osteosarcoma that no pre-amputation biopsy was performed. 
A complete blood count, serum biochemical analysis, regional limb radiography and thoracic radiography were performed prior to anesthesia, and these revealed only mild anemia and dehydration. Though fore limb amputation inhibits natural ambulation which is required for normal breeding process but with the owner's consent, the right thoracic limb was amputated through Mid-humeral amputation. After a complete blood count, serum biochemical analysis, regional limb radiography and thoracic radiography (to evaluate any metastasis of the neoplasia that was not present in this case) and patient health monitoring. Animal was anesthetized and aseptically prepared for amputation of the affected limb. Animal was premedicated with Xylazine $(1 \mathrm{mg} / \mathrm{kg})$ and then intravenous propofol ( $5 \mathrm{mg} / \mathrm{kg}$ ) was administered to induce anesthesia and $2 \%$ isoflurane was used to maintain anesthesia.

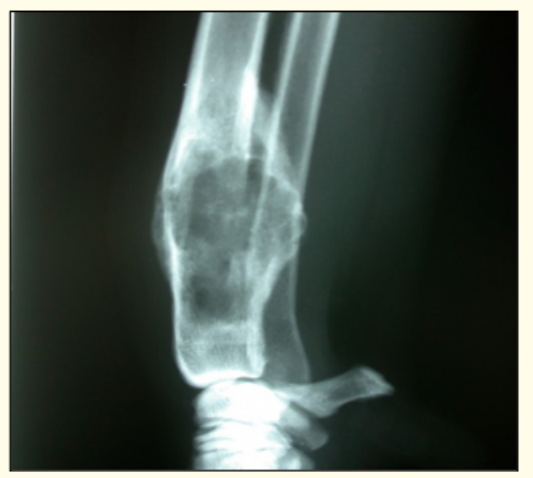

Figure 1: Radiograph of distal radius of deer presenting osteosarcoma. Note the cortical bone destruction, periosteal bone proliferation and soft tissue swelling.

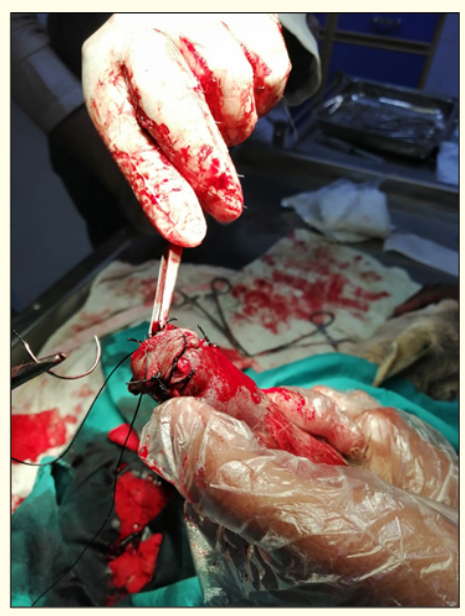

Figure 2: Skin was sutured using simple interrupted sutures using non-absorbable suture material.

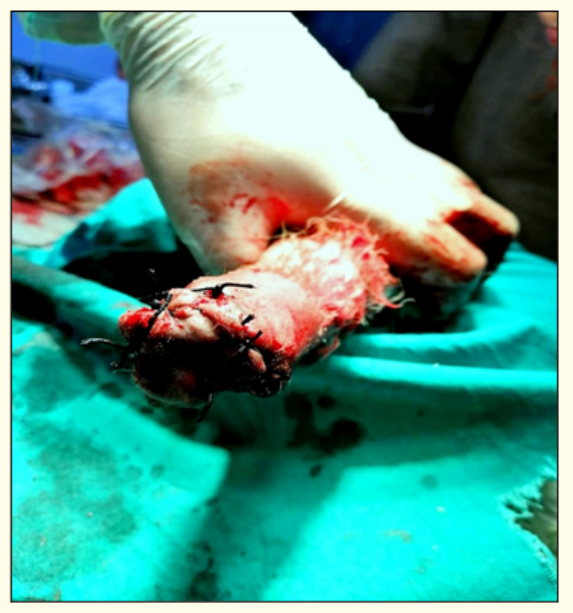

Figure 3: After mid-humeral amputation, chemotherapeutic treatment (carboplatin and doxorubicin) was planned.

Consequently, animal had an uneventful recovery.

A semi roundabout incision was given on the lateral aspect of the limb reaching out from a line through the center of the humerus down to the elbow joint and afterward joined by the comparable incision on the medial aspect of the limb. At that point the skin fold was reflected laterally to uncover the triceps brachii muscle and brachiocephalicus muscle. Cephalic vein was then ligated. Then the common tendon of the triceps brachii and the radial nerve were cut off. At that point the skip fold present medially was reflected to uncover the biceps brachii muscle. Brachial artery and vein were ligated and the Ulnar nerve was cut off proximally. Later on the brachialis, brachiocephalicus and biceps brachii were separated and the shaft of the humerus was uncovered followed by the utilization of hack-saw sharp edge blade to saw off by severing through the shaft of the humerus about $4 \mathrm{~cm}$ over the elbow joint. At that point the closures of the cut off muscles were sutured together utilizing synthetic absorbable suture material over the stump of humerus to give a defensive muscle cushion. The brachialis and biceps brachii muscle were first sutured together over the stump and afterward the brachiocephalicus and triceps muscles. Then the skin folds were sutured by the horizontal mattress suture pattern utilizing the non-absorbable suture material [5].

Animal was well hydrated before and after the surgery and broad-spectrum antibiotics were administered post-operatively.

The removed limb was submitted for histopathological evaluation. When grossly examined radius had an unencapsulated, noncircumscribed and infiltrative neoplastic growth along with destruction of the cortical bone. The swelling around the bone was characterized by the neoplastic proliferation of the surrounding 


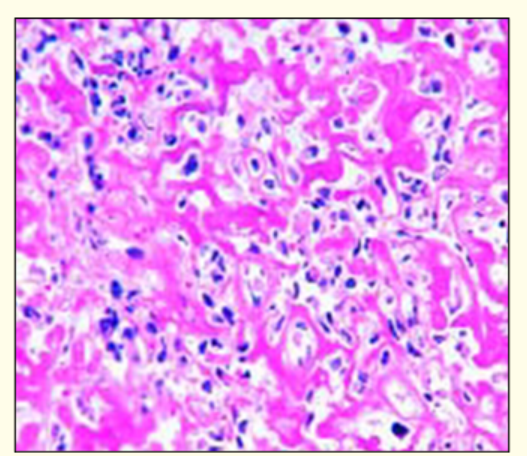

Figure 4: Marked osteoid production with pleomorphic malignant cells incorporation-characteristic of osteosarcoma.

soft tissues i.e. muscles. Histopathology revealed malignant population of the mesenchymal cells that were spindyloid or polygonal in shape. A very marked osteoid production was also observed characteristic of the Osteosarcoma [6].

After limb amputation, deer was administered with Curative Intent Chemotherapy. Chemotherapy protocol included

Carboplatin ( 4 doses) (300 mg/m² every 3 weeks) and Doxorubicin ( 5 doses) (30 mg/m² every 3 weeks).

Later on, at a regular interval of 3 weeks Animal's thoracic and regional limb radiographs were taken regularly to evaluate whether there was any tumor recurrence or metastasis to lungs or other soft tissues. Fortunately, no such condition was observed and animal was doing well.

Osteosarcoma is locally aggressive, causing bone lysis and/or production. Metastasis is common and usually occurs early in the course of disease. Although fewer than $15 \%$ of affected animals have radiographically detectable thoracic metastases at presentation, $90 \%$ die or are euthanized within 1 year of diagnosis because of complications associated with pulmonary metastasis [7]. Improved chances for survival are possible with amputation or limbsparing procedures combined with chemotherapy.

\section{Conclusion}

Although amputation is a major surgery, complications are few if proper technique and good postoperative care are used. The major risks of amputation are blood loss and infection. Local tumor recurrence is quite rare after amputation for osteosarcoma. If ostectomy is performed too close to the tumor, risk exists of leaving tumor cells in the soft tissue or in the medullary canal of the bone in the amputation stump [6].
Another surgical technique which is now being adapted for treatment of bone tumors is Limb Sparing. But Limb sparing is not an appropriate choice of surgery for the treatment of animals with appendicular osteosarcoma.

\section{Limitations of the Study}

The basic reasons for limitation of this technique are the risks associated with this technique such as Tumor recurrence, infection, implant loosening or failure. Furthermore more time and money is required than limb amputation. Be that as it may, the survival result for animals treated with appendage salvage or removal, gave sufficient fundamental chemotherapy is given, is the equivalent [6].

Hence the Limb Amputation is more effective method (less tumor recurrence, cost effective) as compared to limb sparing (Tumor recurrence, risks of implant loosening, infection and require more expertise-only to be performed by an experienced surgeon)

The downside is that this doesn't cure the animal. If no further treatment is administered, the mean survival time is only three or four months. Even with chemotherapy to delay the spread of metastatic disease, life expectancy was only about one year, so the ethical dilemma was whether it was in the animal's best interests to interfere - by amputation or by providing limb-sparing surgery - if it was unlikely to live very long [2].

\section{Bibliography}

1. Brodey Robert S and Wayne H Riser. "7 Canine Osteosarcoma: A Clinicopathologic Study of 194 Cases". Clinical Orthopaedics and Related Research 62 (1969): 54-64.

2. Morris Arianwen. "Managing canine osteosarcoma: limb sparing versus amputation?". Veterinary Record 168.16 (2011): 420-421.

3. Liptak Julius M., et al. "Cortical allograft and endoprosthesis for limb-sparing surgery in dogs with distal radial osteosarcoma: a prospective clinical comparison of two different limbsparing techniques". Veterinary Surgery 35.6 (2006): 518-533.

4. Straw Rodney C and Stephen J Withrow. "Limb-sparing surgery versus amputation for dogs with bone tumors". Veterinary Clinics: Small Animal Practice 26.1 (1996): 135-143.

5. Hickman John and Robert G Walker. "An atlas of veterinary surgery. No. $2^{\text {nd }}$ edition". John Wright and Sons., (1980). 
6. Klein Michael J and Gene P Siegal. "Osteosarcoma: anatomic and histologic variants". American Journal of Clinical Pathology 125.4 (2006): 555-581.

7. Fossum Theressa Welch. "Small Animal Surgery Textbook". Mosby Co., St. Louis, Missouri (2007): 515-520.

8. Shin Hyeok-Soo., et al. "Periarticular histiocytic sarcoma of a thoracic limb in a Rottweiler". Korean Journal of Veterinary Research 58.1 (2018): 57-60.

9. Butt Troy D., et al. "Outcome of limb amputations in wapiti: 13 cases (1995-2001)". The Canadian Veterinary Journal 42.12 (2001): 936-939.

10. Quessada Ana Maria. "Forelimb amputation in a red deer". The Canadian Veterinary Journal 34.7 (1993): 445-446.

\section{Assets from publication with us}

- Prompt Acknowledgement after receiving the article

- Thorough Double blinded peer review

- Rapid Publication

- Issue of Publication Certificate

- High visibility of your Published work

Website: www.actascientific.com/

Submit Article: www.actascientific.com/submission.php Email us: editor@actascientific.com

Contact us: +919182824667 